BULLETIN Bulletin hispanique

HISPANIQUE Université Michel de Montaigne Bordeaux

119-1 | 2017

Autorité et pouvoir dans le théâtre du Siècle d'Or

\title{
Araceli Iravedra (ed.), Políticas Poéticas. De canon y compromiso en la poesía española del siglo XX
}

Iberoamericana, Madrid, Frankfurt, 2013

\section{Claude Le Bigot}

\section{OpenEdition}

\section{Journals}

Édition électronique

URL : http://journals.openedition.org/bulletinhispanique/5007

DOI : 10.4000/bulletinhispanique.5007

ISBN : 979-10-300-0142-6

ISSN : $1775-3821$

Éditeur

Presses universitaires de Bordeaux

Édition imprimée

Date de publication : 15 juin 2017

Pagination : 396-398

ISBN : 979-10-300-0141-9

ISSN : 0007-4640

\section{Référence électronique}

Claude Le Bigot, "Araceli Iravedra (ed.), Políticas Poéticas. De canon y compromiso en la poesía española del siglo XX », Bulletin hispanique [En ligne], 119-1 | 2017, mis en ligne le 15 juin 2017, consulté le 23 septembre 2020. URL : http://journals.openedition.org/bulletinhispanique/5007 ; DOI : https://doi.org/ 10.4000/bulletinhispanique.5007

Ce document a été généré automatiquement le 23 septembre 2020.

Tous droits réservés 


\title{
Araceli Iravedra (ed.), Políticas Poéticas. De canon y compromiso en la poesía española del siglo XX
}

Iberoamericana, Madrid, Frankfurt, 2013

\author{
Claude Le Bigot
}

\section{RÉFÉRENCE}

Araceli Iravedra (ed.), Políticas Poéticas. De canon y compromiso en la poesía española del siglo XX, Madrid, Frankfurt, Iberoamericana, Vervuet, 2013, 260 p.

Cet ouvrage collectif dirigé par Araceli Iravedra, Professeure de littérature espagnole à l'université d'Oviedo, malgré ses dimensions modestes, est une véritable somme qui aurait pu porter le titre de « un siècle d'engagement de la poésie espagnole ». En effet, il balaie tout le siècle depuis le modernisme jusqu'aux premières années du XXI ${ }^{\mathrm{e}}$, pour problématiser une notion dont on ne peut pas faire l'économie : l'engagement de la littérature, réduite ici à la poésie, surtout dans un pays dont on sait qu'elle y a occupé une place privilégiée dans la lutte contre le discours officiel du franquisme. Ce livre s'impose par sa cohérence, qui tient en partie au nombre réduit des contributeurs qui ont pu ainsi disposer d'un large espace (environ 40/50 pages chacun) pour exposer leurs idées. Le plan général couvre les périodes clés où s'exprime une forme d'engagement spécifique : le Modernisme, en tant que rupture esthétique, l'avant-garde et la Révolution jusqu'à la veille de la guerre civile, la subjectivité de l'engagement chez les poètes exilés ou condamnés par le franquisme, l'essor et l'hégémonie de la poésie sociale, les nouveaux questionnements de la conscience civique dans la poésie de l'Espagne démocratique. Chacune de ces contributions pourrait illustrer un chapitre d'une monographie où la notion d'engagement est paradoxalement perçue à travers le prisme d'une possible forme canonique. Ce cadre est pensé comme une authentique provocation, d'une redoutable fécondité cependant, dès lors que l'engagement tend à 
occuper une place centrale dans le champ de la littérature. Un tel positionnement a le mérite de dépasser les dichotomies habituelles: poésie pure vs poésie politique, intimité vs histoire, tradition vs modernité, forme vs contenu; mais chaque auteur partage un tronc commun: pas de canon esthétique qui ne soit le produit d'une idéologie. Du point de vue heuristique, les auteurs s'interrogent sur l'intérêt et la pertinence de la notion de canon et plaident pour un statut fluctuant du concept, puisque la reconnaissance d'une forme hégémonique ne signifie nullement la fixation définitive d'une pratique esthétique.

2 La contribution qui ouvre l'ouvrage, due à Juan Carlos Rodríguez, peut surprendre puisqu'elle consiste à voir dans le modernisme un premier glissement vers l'engagement de la littérature. Thèse hautement paradoxale, puisque nos habitudes culturelles et la doxa véhiculée par une certaine histoire littéraire consistent à voir dans la quête d'un langage épuré la seule voie d'accès à l'Absolu. J. C. Rodríguez va inverser cette perspective, puisque toute l'œuvre poétique de Juan Ramón Jiménez tend à montrer l'impossibilité d'une écriture autonome et suffisante, toute prétendue transcendance finissant par se heurter aux contingences qui prédéterminent la volonté d'écrire. Si engagement il y a dans la Modernisme, il pourrait tenir dans la manière dont Juan Ramón assigne à la poésie un savoir déceptif, dont le fondement irréductible est l'opacité du monde. C'est en s'appuyant sur cette négativité que Juan Ramón Jiménez entretient, selon J. C. Rodríguez, l'ascèse poétique trop souvent confondue avec un idéalisme absolu et inutile.

3 Après une remise en cause assez sévère des formes canoniques, théorisées par Harold Bloom dont les attaches avec l'idéologie bourgeoise du sujet libre reviennent d'avance à renoncer à toute explication sur les raisons de la poésie engagée, Miguel Angel García montre que les tenants de la poésie pure et des avant-gardes obéissent à des idéologies antagonistes et pas forcément - comme on le croit trop souvent - politisées. Cette politisation qui dans le cas de la poésie engagée revient à instrumentaliser l'activité littéraire, ne prend véritablement forme qu'au cours de l'année 1933, où un certain nombre de poètes (Alberti, Prados, Plá y Beltrán, José María Morón) conçoivent leur tâche comme un "impétueux devoir moral». Mais le débat poésie pure vs poésie impure, ne recoupe pas uniformément la coupure art désintéressé vs art engagé: l'impureté dont parlait Pablo Neruda dans son manifeste de 1933 signifiait plus simplement restaurer l'humain dans toutes ses dimensions, déplaçant en quelque sorte le paradigme canonique.

4 Luis Bagué adopte un autre angle d'attaque pour observer les inflexions de la poésie engagée, choisissant délibérément d'accrocher le discours intimiste au témoignage social chez M. Hernández, Max Aub et León Felipe. S'il est vrai que chacun de ces trois poètes publie dans l'immédiat après-guerre civile, soit depuis l'exil, soit depuis le confinement carcéral ou concentrationnaire, tous trois partagent la même situation de vaincus ; or, la sphère privée à laquelle se rattache le discours autobiographique dans ces trois exemples, n'en est pas moins perméable à ce qui constitue la tragédie collective d'une défaite injuste, avec pour horizon une nouvelle dialectique qui puisse faire de l'expiation une rédemption, une expérience qui, pour les poètes de l'exil ou ceux de l'exil intérieur, allait configurer le canon de la diaspora.

Quel projet idéologique et esthétique y a-t-il derrière la poésie sociale des années cinquante-65 en Espagne? Pour répondre à cette question Laura Scarano s'appuie sur les déclarations théoriques, poèmes programmatiques et arts poétiques, soit un corpus 
nommé « autopoéticas del compromiso ", chez Blas de Otero, Gabriel Celaya, José Hierro. Malgré une étude très détaillée de ces trois auteurs, la mise en évidence du canon de la poésie sociale est sans doute un peu biaisée, faute de s'appuyer sur l'aboutissement de positions théoriques dans une praxis; disons simplement, l'analyse de la mise en discours. Scarano est plus convaincante quand il s'agit d'aborder la poésie de Blas de Otero, d'autant plus que son ultime étape (Historias fingidas y verdaderas et Hojas de Madrid con La galerna) vient étayer la nécessaire osmose entre trajectoire individuelle et projet collectif. C'est à ce moment-là que le poète gagne en profondeur et en originalité et que, in fine, le discours poétique parvient à modifier le regard qu'un lecteur peut porter sur un ordre social contestable. En abordant l'œuvre de ces trois poètes majeurs. L. Scarano voit dans le rapport poésie, "savoir et/ou communication", non pas une dichotomie, mais le couplage qui fonde la pertinence de la poésie sociale, et de ce point de vue c'est sans doute José Hierro qui offre l'œuvre la plus novatrice en matière de témoignage, parce que le témoignage n'est plus perçu seulement dans sa dimension perlocutoire ou performative, mais comme une fonctionnalité décisive dans l'émergence d'une nouvelle esthétique.

Araceli Iravedra traite la période la plus récente, pour observer le renouveau de la poésie engagée depuis que l'Espagne est devenue un pays démocratique. Ce qui change significativement les conditions d'existence du discours critique, qui s'était développé sous la dictature franquiste. Certes, les motifs de mécontentements au plan social n'ont pas disparu, mais ils se sont tournés vers des objets transnationaux (méfaits de la globalisation, inégalités croissantes, conséquences d'un capitalisme débridé). Dans ce nouveau contexte, la poésie peut-elle encore tenir un discours critique crédible ? Plutôt que de changer le monde, la poésie pense pouvoir agir sur le sujet; car ce qu'il convient de préserver, c'est la liaison privé/public, que le discours libéral entend régenter et séparer de la manière la plus brutale. Les poètes invoqués ont tous collaboré à des degrés divers à des collectifs (Alicia bajo Cero, Voces del Extremo, Unión de Escritores Valencianos, tels Jorge Riechmann, Antonio Orihuela, Isabel Pérez Montalbán, David González, Enrique Falcón, Antonio Méndez Rubio). On peut toutefois se demander si le retour du réalisme dans la poésie espagnole, qui est d'ailleurs une réaction contre la "privatisation de la littérature » dont parle José Carlos Mainer, est suffisamment puissant pour installer un modèle culturel dominant et conforter un nouveau canon de l'engagement, qui reste tout de même attaché au "principe de réalité ». Ce qui est déterminant chez les nouveaux poètes engagés étudiés par Araceli Iravedra est leur volonté de ne pas être récupérés; cette forme d'insoumission conduit-elle au renouvellement du canon de l'engagement? Telle est la problématique de cet ouvrage ambitieux au plan théorique, et qui est une référence incontournable dans toute réflexion sur les nouvelles formes de l'engagement littéraire. 


\section{AUTEURS}

\section{CLAUDE LE BIGOT}

Université de Rennes 2 